\title{
Severe Heart Failure and Large Left Ventricular Thrombus Following ACUTe Myocardial Infarction
}

\author{
Ankitkumar K. Patel MD, MPH, Scott Silvestry, MD, and Paul J. Mather, MD
}

\section{Case Presentation}

A 58 year-old man who recently underwent a left superficial femoral artery thrombectomy presented with a three-day history of worsening exertional dyspnea and bilateral pedal edema. His past medical history is significant for coronary artery disease, myocardial infarction, and insulin dependent diabetes mellitus. The patient initially presented to an outside hospital where he developed ventricular tachycardia that warranted cardioversion three times. Initial electrocardiogram showed inferior lead ST segment elevations and lateral lead ST depression. The patient underwent a cardiac catheterization that showed triple vessel disease with total occlusion of the RCA, $90 \%$ occlusion of the

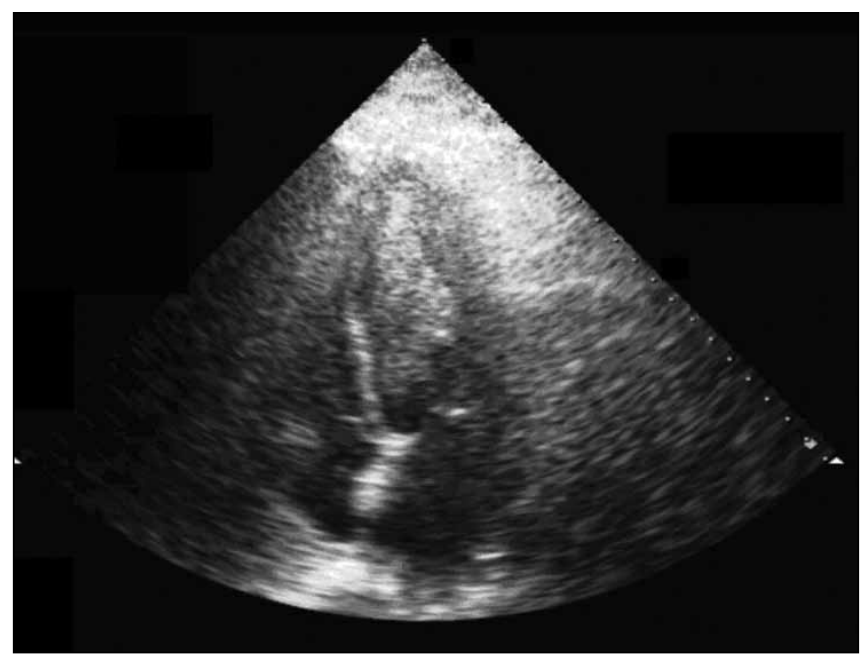

Figure 1. Transthoracic echocardiogram shows a large mobile thrombus occupying $60 \%$ of the left ventricular cavity.

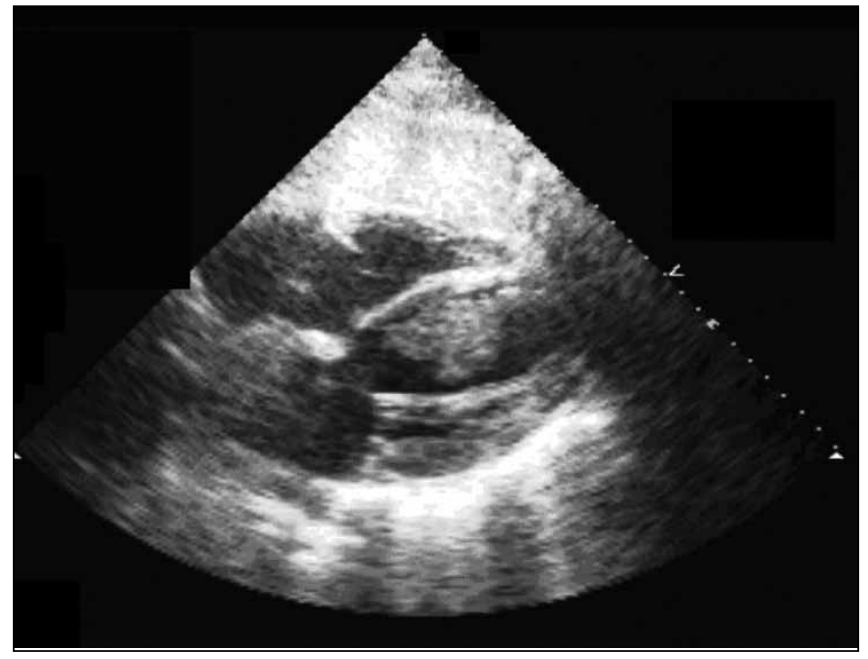

Figure 2. In another view, the echocardiogram shows a large mobile thrombus within the left ventricular cavity.
LAD, and $50 \%$ to $60 \%$ occlusion of left circumflex. The patient had an echocardiogram (Figures 1 and 2) that showed severe left ventricular dysfunction with an ejection fraction of $10 \%$ and a large mobile thrombus occupying $60 \%$ of the left ventricular cavity and was diagnosed with a dilated cardiomyopathy. The patient underwent left ventricular thrombectomy (Figure 3), coronary artery bypass graft (i.e., a saphenous vein graft to LAD) and installation of a left ventricular assist device. The patient currently is doing well and is awaiting heart transplantation.

\section{Discussion}

An increased incidence of thromboembolism is seen in patients with left ventricular systolic dysfunction following myocardial infarction (MI). ${ }^{1}$ Following an acute MI, the formation of a left ventricular (LV) thrombus is a significant complication. LV thrombus occurs in up to one-third of patients with anterior wall MI and is much more frequent in patients with a large anterior MI and subsequent heart failure. ${ }^{2}$ The vast majority of LV thrombi are of the immobile mural type; unfortunately for our patient, he had the more rare mobile type which has a higher risk of embolism. ${ }^{3} \mathrm{LV}$ thrombus has been associated with

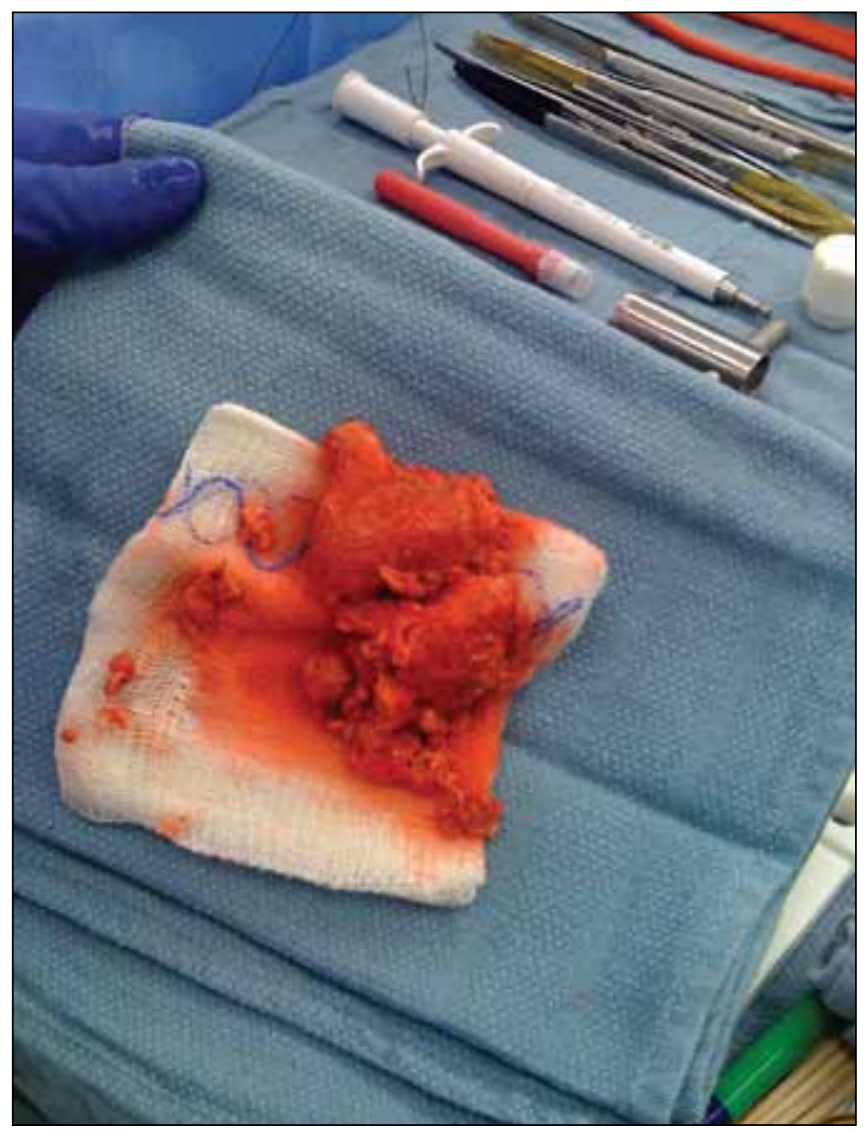

Figure 1. Post-operative specimen of left ventricular thrombetomy. 
dilated cardiomyopathy which leads to increased morbidity and mortality. ${ }^{4}$ The mechanism for the development of a mobile thrombus is not well understood. Moran et al hypothesize that an apical infarction involves the detachment of one end of the trabecula of the left ventricle which acts as a nidus for thrombosis. Prompt surgery is warranted once a mobile thrombus is identified. ${ }^{6}$ Left ventricular thrombectomy and coronary artery bypass graft surgery after an acute MI have increased risks. In order to improve the clinical status of our patient, he had a left assist device inserted during the surgery to help reduce the LV workload and provide a bridge to cardiac transplantation.

\section{References}

1. Motto A, Ballo P. Two- and three-dimensional echocardiograhic imaging of massive left ventricular thrombosis complicating fatal heart failure. Journal of Cardiol 2007; 116(1): e10-12.

2. Visser CA, Kan G, Lie KI, et al. Left ventricular thrombus following acute myocardial infarction: A prospective serial Echocardiographic study of 96 patients. Europ Heart Journal 1983; 4: 333-337.
3. Minami H, Asada T, Gan K. Surgical removal of left ventricular ball-like thrombus following large transmural acute myocardial infarction using the infarction exclusion technique. Circulation Journal 2008; 72: 1547-1549.

4. Mazzone M, La Sala M, Portale G, et al. Review of dilated cardiomyopathies. Dilated cardiomyopathies and altered prothrombotic state: a point of view of the literature. Panminerva Med 2005; 47(3):157-167.

5. Moran JM, Michaelis LL. Surgery for the complications of myocardial infarction. New York. Grune \& Strutton; 1980; 315-317.

6. Moran JM, Asinger RW, Mikell FL, et al. Embolic potential of left ventricular thrombi detected by two-dimensional echocardiography. Circulation 1984; 70: 333-337. 